\title{
Identification of aldolase $A$ as a potential diagnostic biomarker for colorectal cancer based on proteomic analysis using formalin-fixed paraffin-embedded tissue
}

\author{
Tetsushi Yamamoto $^{1}$ - Mitsuhiro Kudo ${ }^{2}$ - Wei-Xia Peng ${ }^{2} \cdot$ Hideyuki Takata ${ }^{2,3}$. \\ Hideki Takakura $^{1} \cdot$ Kiyoshi Teduka $^{2} \cdot$ Takenori Fujii $^{2} \cdot$ Kuniko Mitamura $^{1}$. \\ Atsushi Taga ${ }^{1} \cdot$ Eiji Uchida $^{3} \cdot$ Zenya Naito $^{2}$
}

Received: 24 March 2016 / Accepted: 15 July 2016 / Published online: 28 July 2016

(C) The Author(s) 2016. This article is published with open access at Springerlink.com

\begin{abstract}
Colorectal cancer (CRC) is one of the most common cancers worldwide, and many patients are already at an advanced stage when they are diagnosed. Therefore, novel biomarkers for early detection of colorectal cancer are required. In this study, we performed a global shotgun proteomic analysis using formalin-fixed and paraffin-embedded (FFPE) CRC tissue. We identified 84 candidate proteins whose expression levels were differentially expressed in cancer and non-cancer regions. A label-free semiquantitative method based on spectral counting and gene ontology (GO) analysis led to a total of 21 candidate proteins that could potentially be detected in blood. Validation studies revealed cyclophilin A, annexin A2, and aldolase A mRNA and protein expression levels were significantly higher in cancer regions than in non-cancer regions. Moreover, an in vitro study showed that secretion of aldolase A into the culture medium was clearly suppressed in CRC cells compared to normal colon epithelium. These findings suggest that decreased aldolase A in blood may be a novel biomarker for the early detection of CRC.
\end{abstract}

Keywords Colorectal cancer · Shotgun proteomics . Formalin-fixed paraffin-embedded tissue $\cdot$ Aldolase A

\section{Zenya Naito}

naito@nms.ac.jp

1 Pathological and Biomolecule Analyses Laboratory, Faculty of Pharmacy, Kindai University, Osaka, Japan

2 Department of Integrated Diagnostic Pathology, Nippon Medical School, 1-1-5 Sendagi, Bunkyo-ku, Tokyo 113-8602, Japan

3 Departments of Gastrointestinal Hepato Biliary Pancreatic Surgery, Nippon Medical School, Tokyo, Japan

\section{Introduction}

Colorectal cancer (CRC) is one of the most common cancers worldwide. If the tumor is limited to the mucosa or submucosa, CRC can be completely cured by endoscopic or surgical therapy; however, many patients are already at an advanced stage when they are diagnosed. Therefore, an early detection method is needed. Diagnostic blood tests based on detection of carcinoembryonic antigen (CEA) are now widely used for CRC, but the sensitivity of this biomarker in early-stage cancer is only $5-10 \%[1,2]$. The identification of novel candidate molecules that are secreted by cancer cells may lead to the development of early detection methods and improved prognosis.

Formalin-fixed and paraffin-embedded (FFPE) tissues are archived in hospitals, together with detailed clinical information such as disease history, clinical examination results, and drug responses. FFPE tissues are routinely used in the diagnosis and research of diseases, including cancers, by conventional staining, immunohistochemistry (IHC), and in situ hybridization [3-5]. Previously, FFPE tissues were considered unsuitable for proteomic analysis. However, methods to isolate protein from FFPE tissues for proteomic analysis have been developed, and various FFPE tissues have been used in proteomic studies [6-17]. Thus, archived FFPE tissues may be useful for identifying new biomarkers.

In this study, we performed shotgun liquid chromatography (LC)/mass spectrometry (MS)-based global proteomic analysis using proteins from FFPE CRC tissue to identify proteins whose expression levels are modified in cancer regions. We identified aldolase A as a candidate protein with potential as a novel biomarker for early detection of CRC. 


\section{Materials and methods}

\section{Materials}

The following materials were purchased from Wako Pure Chemical Industries (Osaka, Japan): guanidine hydrochloride, DTT, Tris, tris (2-carboxyethyl) phosphine hydrochloride (TCEP), and iodoacetamide (IAA). All other chemicals and reagents were purchased from Sigma Chemical Corp. (St. Louis, MO, USA).

\section{Patients and tissue specimens}

The tissues used in this study were from 45 patients who underwent surgical resection for CRC at Nippon Medical School Hospital between October 2007 and August 2014. None of the patients received chemotherapy or radiation therapy prior to surgery and none had inflammatory colorectal disease such as colitis or infectious diseases. The pathological diagnosis and clinicopathological stage were determined according to the criteria of the World Health Organization [18]. After a histopathological analysis, 10 of these 45 cases were selected for proteomic analysis. Immunohistochemical analyses were performed on all 45 cases. Paraffin-embedded specimens were prepared for proteomic and immunohistochemical analyses. This study was carried out in accordance with the principles embodied in the Declaration of Helsinki, 2013, and the Japanese Society of Pathology Ethics Committee. Informed consent for the use of colorectal tissues was obtained from all patients.

\section{Protein extraction from FFPE tissue}

FFPE CRC tissues from ten patients were used for proteomic analysis. Pathological and clinical information are shown in Table 1. Following histological examination of hematoxylin and eosin (H\&E) sections, we separated the cancer regions from the non-cancer regions, which maintained normal structures. Sections $(10 \mu \mathrm{m})$ were deparaffinized in xylene and rehydrated through a series of graded alcohols $(100,90,80$, and $70 \%$ ). After staining with Mayer's hematoxylin for $5 \mathrm{~min}$, cancer and non-cancer regions were manually dissected under a microscope (Fig. 1). Proteins were extracted from both cancer and non-cancer regions using lysis buffer (6 M guanidine- $\mathrm{HCl}, 40 \mathrm{mM}$ Tris- $\mathrm{HCl} \mathrm{pH} 8.2$, and $65 \mathrm{mM}$ DTT) according to a previous report [17]. Protein concentration was measured by the Bradford method.

\section{In-solution trypsin digestion}

A gel-free digestion was performed according to the protocol described by Bluemlein et al. [19], with slight modifications. Briefly, $10 \mu \mathrm{g}$ of protein was extracted from each sample, reduced with $45 \mathrm{mM}$ DTT and $20 \mathrm{mM}$ TCEP, and then alkylated using $100 \mathrm{mM}$ IAA. After alkylation, samples were digested with proteomics-grade trypsin (Agilent Technologies Inc., Santa Clara, CA, USA) at $37^{\circ} \mathrm{C}$ for $24 \mathrm{~h}$. Digests were purified using PepClean C-18 Spin Columns (Thermo, Rockford, IL, USA) according to the manufacturer's protocol.

\section{LC-MS/MS analysis for protein identification}

Approximately $2 \mu \mathrm{g}$ of purified peptide samples were injected onto a peptide L-trap column (Chemicals Evaluation and Research Institute, Tokyo, Japan) using an HTS PAL autosampler (CTC Analytics, Zwingen, Switzerland) and further separated thorough an Advance-nano UHPLC (AMR Inc., Tokyo, Japan) using a reverse-phase C18-column (Zaplous column $\alpha, 3-\mu \mathrm{m}$ diameter gel particles and $100 \AA$ pore size, $0.1 \times 150 \mathrm{~mm}$; AMR). The mobile phase consisted of solution A ( $0.1 \%$ formic acid in water) and solution B (acetonitrile). The flow rate was $500 \mathrm{~nL} / \mathrm{min}$, with a concentration gradient of acetonitrile from $5 \% \mathrm{~B}$ to $35 \% \mathrm{~B}$ over $120 \mathrm{~min}$. Gradient-eluted peptides were analyzed using an amaZon ETD ion-trap mass spectrometer (Bruker Daltonics, Billerica, MA, USA). Data were acquired in a data-dependent manner, in which MS/MS fragmentation was performed on the ten most intense peaks of every full MS scan.

All MS/MS spectra data were searched against the SwissProt Homo sapiens database with Mascot (version_2.3.01; Matrix Science, London, UK). Search criteria were as follows: enzyme, trypsin; allowance of up to two missed cleavage peptides; mass tolerance $\pm 0.5 \mathrm{Da}$ and MS/ MS tolerance $\pm 0.5 \mathrm{Da}$; and modifications of cysteine carbamidomethylation, methionine oxidation, and $\mathrm{N}$-formylation including formyl (K), formyl (R), and formyl (N terminus).

\section{Spectral counting analysis of identified proteins}

To compare protein expression across all tissue samples, we used the spectral counting method. In this analysis, when not specific spectral peak could be identified, the expression level of that protein was taken as zero, as described in a previous report [20]. Fold-changes of expressed proteins in the base 2 logarithmic scale were calculated with Rsc based upon spectral counting [21]. Relative amounts of identified proteins were also calculated with the normalized spectral abundance factor (NSAF) [22]. Candidate proteins with modified expression levels in the cancer region were chosen to ensure that the Rsc satisfied $>1$ or $<-1$, which corresponded to fold changes of $>2$ or $<0.5$.

\section{Bioinformatics}

Functional annotations for the identified proteins with modified expression levels in the cancer region were processed using the Database for annotation, visualization, and 
Table 1 Clinical and pathological data of patients with CRC that contributed tissues for proteomic analysis

\begin{tabular}{|c|c|c|c|c|c|c|c|}
\hline \multirow[t]{2}{*}{ Patient No. } & \multirow[t]{2}{*}{ Age (year) } & \multirow[t]{2}{*}{ Gender } & \multirow[t]{2}{*}{ pTNM } & \multirow[t]{2}{*}{ pStaging } & \multirow[t]{2}{*}{ Tumor location } & \multicolumn{2}{|c|}{$\begin{array}{l}\text { Number of identified } \\
\text { proteins }\end{array}$} \\
\hline & & & & & & $\begin{array}{l}\text { Cancer } \\
\text { region }\end{array}$ & $\begin{array}{l}\text { Non-cancer } \\
\text { region }\end{array}$ \\
\hline 1 & 63 & M & T3N0M0 & Stage IIA & S & 49 & 26 \\
\hline 2 & 53 & M & T3N0M0 & Stage IIA & $\mathrm{S}$ & 38 & 38 \\
\hline 3 & 77 & $\mathrm{~F}$ & T3N0M0 & Stage IIA & $\mathrm{S}$ & 50 & 34 \\
\hline 4 & 53 & M & T3N0M0 & Stage IIA & $\mathrm{S}$ & 69 & 52 \\
\hline 5 & 76 & $\mathrm{~F}$ & T3N0M0 & Stage IIA & $\mathrm{S}$ & 52 & 22 \\
\hline 6 & 51 & M & T3N0M0 & Stage IIA & A & 22 & 4 \\
\hline 7 & 67 & $\mathrm{~F}$ & TisN0M0 & Stage 0 & A & 44 & 28 \\
\hline 8 & 52 & M & T3N0M0 & Stage IIA & $\mathrm{Ra}$ & 52 & 50 \\
\hline 9 & 69 & M & T3N0M0 & Stage IIA & $\mathrm{T}$ & 65 & 16 \\
\hline 10 & 69 & $\mathrm{~F}$ & T3N0M0 & Stage IIA & $\mathrm{S}$ & 19 & 35 \\
\hline
\end{tabular}

$M$ male, $F$ female, $A$ ascending colon, $T$ transverse colon, $S$ sigmoid colon, $R a$ upper rectum integrated discovery (DAVID) version 6.7 (http://david. abcc.ncifcrf.gov/home.jsp) [23-25].

\section{Quantitative RT-PCR}

Total RNA was extracted from FFPE CRC tissue with the RNeasy FFPE Kit (QIAGEN, Valencia, CA, USA) and from CRC cell lines with the GenElute Mammalian Total RNA Miniprep Kit (Sigma). cDNA was synthesized using the SuperScript VILO cDNA Synthesis Kit for FFPE tissue and the High Capacity cDNA Reverse Transcription kit for the CRC cell line according to the manufacturer's protocols (Life Technologies Japan, Tokyo, Japan). To measure expression of aldolase A, cyclophilin A, and annexin A2 mRNA, quantitative reverse transcription PCR (qRT-PCR) was performed with the 7500 system (Applied Biosystems, Foster City, CA, USA). Primers and TaqMan probes for aldolase A (Hs00765620_m1), cyclophilin A (Hs04194521_s1), annexin A2 (Hs00743063_s1), and 18S rRNA (Hs03928990_g1) were used with the TaqMan Gene Expression Assay. qRTPCR results were expressed relative to an internal standard concentration as a ratio of target/18S rRNA. Gene expression was measured in triplicate.

\section{Immunohistochemistry}

FFPE CRC tissues from 46 patients were used for a validation analysis. Pathological and clinical information are
Fig. 1 Manual dissection of FFPE colon tissue.

Representative image shows noncancer and cancer regions before (a and b, respectively) and after (c and $\mathbf{d}$, respectively) dissection. Sections were stained with hematoxylin a

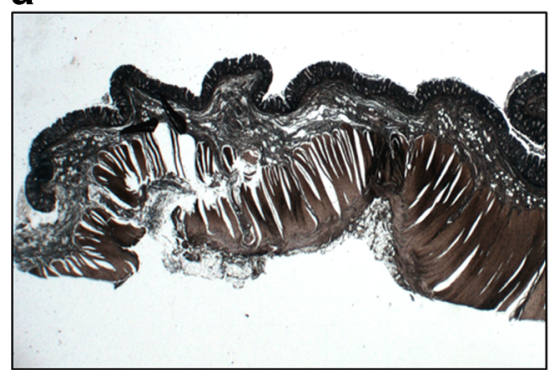

C

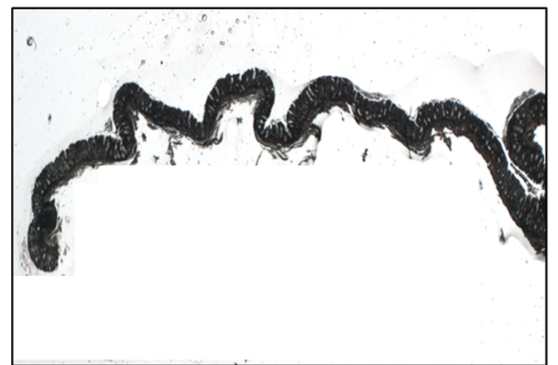

b

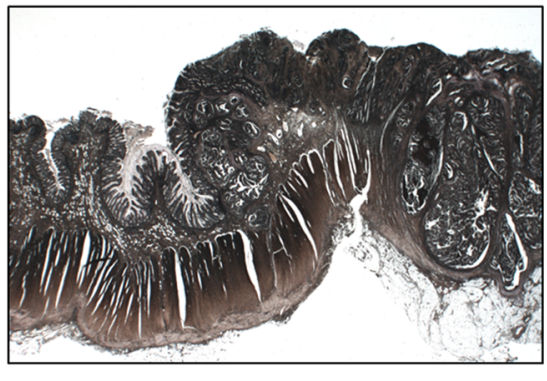

d

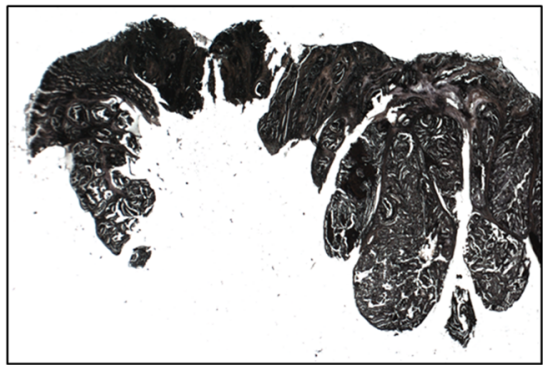


Table 2 Clinical and pathological data of patients with CRC that contributed tissues for immunohistochemical analysis

\begin{tabular}{|c|c|c|c|c|c|}
\hline Patient No. & $\begin{array}{l}\text { Age } \\
\text { (year) }\end{array}$ & Gender & pTNM & pStaging & $\begin{array}{l}\text { Tumor } \\
\text { location }\end{array}$ \\
\hline 1 & 67 & F & TisN0M0 & Stage 0 & $\mathrm{~A}$ \\
\hline 2 & 66 & F & TisN0M0 & Stage 0 & $\mathrm{D}$ \\
\hline 3 & 61 & F & TisN0M0 & Stage 0 & $\mathrm{~S}$ \\
\hline 4 & 70 & M & TisN0M0 & Stage 0 & $\mathrm{~T}$ \\
\hline 5 & 64 & F & TisN0M0 & Stage 0 & $\mathrm{~T}$ \\
\hline 6 & 63 & M & T2N0M0 & Stage I & $\mathrm{Rb}$ \\
\hline 7 & 58 & F & T2N0M0 & Stage I & $\mathrm{Rb}$ \\
\hline 8 & 45 & F & T2N0M0 & Stage I & $\mathrm{Rb}$ \\
\hline 9 & 67 & F & T2N0M0 & Stage I & $\mathrm{Ra}$ \\
\hline 10 & 87 & F & T2N0M0 & Stage I & $\mathrm{Ra}$ \\
\hline 11 & 67 & F & T2N0M0 & Stage I & $\mathrm{S}$ \\
\hline 12 & 70 & M & T1N0M0 & Stage I & $\mathrm{S}$ \\
\hline 13 & 75 & $\mathrm{~F}$ & T1N0M0 & Stage I & $\mathrm{T}$ \\
\hline 14 & 82 & M & T1N0M0 & Stage I & $\mathrm{T}$ \\
\hline 15 & 63 & M & T3N0M0 & Stage IIA & $\mathrm{S}$ \\
\hline 16 & 53 & M & T3N0M0 & Stage IIA & $\mathrm{S}$ \\
\hline 17 & 77 & F & T3N0M0 & Stage IIA & $\mathrm{S}$ \\
\hline 18 & 53 & M & T3N0M0 & Stage IIA & $\mathrm{S}$ \\
\hline 19 & 76 & F & T3N0M0 & Stage IIA & $\mathrm{S}$ \\
\hline 20 & 51 & M & T3N0M0 & Stage IIA & A \\
\hline 21 & 52 & M & T3N0M0 & Stage IIA & $\mathrm{Ra}$ \\
\hline 22 & 69 & M & T3N0M0 & Stage IIA & $\mathrm{T}$ \\
\hline 23 & 69 & F & T3N0M0 & Stage IIA & $\mathrm{S}$ \\
\hline 24 & 92 & M & T3N0M0 & Stage IIA & $\mathrm{T}$ \\
\hline 25 & 64 & M & T2N1aM0 & Stage IIIA & $\mathrm{Ra}$ \\
\hline 26 & 73 & F & $\mathrm{T} 2 \mathrm{~N} 1 \mathrm{bM} 0$ & Stage IIIA & $\mathrm{Ra}$ \\
\hline 27 & 64 & M & T3N1aM0 & Stage IIIB & $\mathrm{Ra}$ \\
\hline 28 & 50 & F & T3N1bM0 & Stage IIIB & $\mathrm{S}$ \\
\hline 29 & 51 & M & T3N1bM0 & Stage IIIB & A \\
\hline 30 & 64 & M & T3N1aM0 & Stage IIIB & $\mathrm{S}$ \\
\hline 31 & 64 & $\mathrm{~F}$ & T3N1bM0 & Stage IIIB & $\mathrm{Ra}$ \\
\hline 32 & 78 & $\mathrm{~F}$ & T3N1aM0 & Stage IIIB & A \\
\hline 33 & 72 & F & T4aN1AM0 & Stage IIIB & $\mathrm{C}$ \\
\hline 34 & 52 & M & T3N1bM0 & Stage IIIB & $\mathrm{Ra}$ \\
\hline 35 & 85 & $\mathrm{~F}$ & T4bN1aM0 & Stage IIIC & $\mathrm{A}$ \\
\hline 36 & 70 & M & T4aN2aM0 & Stage IIIC & $\mathrm{S}$ \\
\hline 37 & 81 & F & T4aN0M1a & Stage IVA & $\mathrm{T}$ \\
\hline 38 & 71 & F & T4aN2aM1a & Stage IVA & A \\
\hline 39 & 49 & M & T3N2aM1a & Stage IVA & $\mathrm{C}$ \\
\hline 40 & 78 & M & T3N1bM1a & Stage IVA & $\mathrm{Ra}$ \\
\hline 41 & 77 & M & T4aN2aM1a & Stage IVA & $\mathrm{T}$ \\
\hline 42 & 57 & M & $\mathrm{T} 3 \mathrm{~N} 2 \mathrm{bM} 1 \mathrm{~b}$ & Stage IVB & $\mathrm{Ra}$ \\
\hline 43 & 72 & $\mathrm{~F}$ & T4aN0M1b & Stage IVB & $\mathrm{D}$ \\
\hline 44 & 71 & $\mathrm{~F}$ & $\mathrm{~T} 4 \mathrm{bN} 2 \mathrm{bM} 1 \mathrm{~b}$ & Stage IVB & $\mathrm{C}$ \\
\hline 45 & 70 & M & T3N0M1b & Stage IVB & $\mathrm{S}$ \\
\hline
\end{tabular}

$M$ male, $F$ female, $A$ ascending colon, $T$ transverse colon, $S$ sigmoid colon, $R a$ upper rectum, $R b$ lower rectum, $D$ descending colon, $C$ cecum

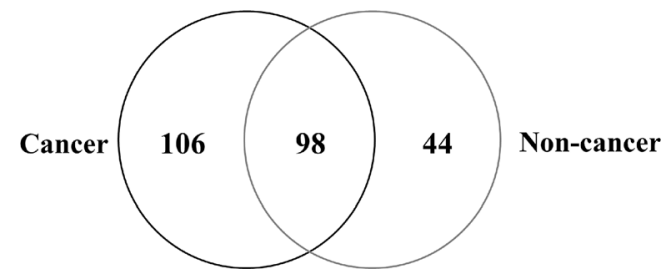

Fig. 2 Venn map of proteins identified from FFPE colorectal cancer tissue. We identified 204 proteins in the cancer regions and 142 in the non-cancer regions

shown in Table 2. Paraffin-embedded tissue sections (3 $\mu \mathrm{m})$ were subjected to immunostaining using a Histofine Simple Stain MAX-PO (R) kit (Nichirei, Tokyo, Japan) for identifying aldolase A, annexin A2, and cyclophilin A. After deparaffinization, sections were pretreated in an autoclave at $121{ }^{\circ} \mathrm{C}$ for $15 \mathrm{~min}$ in $10 \mathrm{mM}$ citrate buffer ( $\mathrm{pH}$ 6.0) for cyclophilin A. Endogenous peroxidase activity was blocked by incubation for $30 \mathrm{~min}$ with $0.3 \%$ hydrogen peroxide in methanol. Tissue sections were then incubated with the anti-ALDOA antibody (1:150 dilution; Atlas Antibodies, Stockholm, Sweden) for aldolase A, anti-cyclophilin A antibody (1:150 dilution; Novus Biologicals, Littleton, CO, USA), or antiannexin A2 antibody (1:400 dilution; Cell Signaling Technology, Inc., Danvers, MA, USA) in phosphatebuffered saline containing $1 \%$ bovine serum albumin for $16 \mathrm{~h}$ at $4{ }^{\circ} \mathrm{C}$. Bound antibodies were detected with the Simple Stain MAX-PO (R) with diaminobenzidine tetrahydrochloride as the substrate. Sections were then counterstained with Mayer's hematoxylin. Two investigators (TY and HT) evaluated all the sections separately in a blinded manner. Sections were scored for both intensity ( 0 , no stain; 1 , weak; 2 , moderate; and 3 , strong) and percentage of epithelial cells that stained positive $(0,0$ $5 \% ; 1,6-25 \% ; 2,26-50 \% ; 3,51-75 \%$; and 4, 76$100 \%)$. Scores were derived from the sum of the intensity and percentage of immunoreactive cells [15].

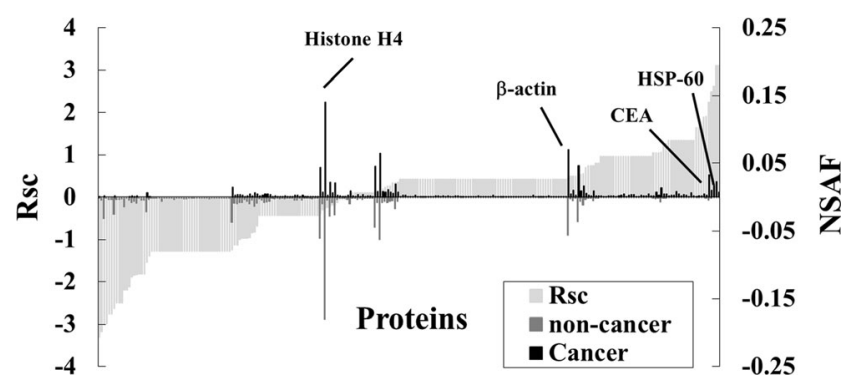

Fig. 3 Semiquantitative comparison of proteins identified in FFPE colon tissue. Rsc and normalized spectral abundance factor (NSAF) values calculated for the proteins identified ( $x$-axis). Comparison of protein expression in cancer versus non-cancer tissue. Proteins highly expressed in either cancer or non-cancer regions were plotted near the right or left side of the $x$-axis 
Table 3 Differentially expressed proteins in cancer region of CRC samples

\begin{tabular}{|c|c|c|c|c|c|c|}
\hline \multirow[t]{2}{*}{ No. } & \multirow[t]{2}{*}{ ID } & \multirow[t]{2}{*}{ Accession number and description } & \multirow{2}{*}{$\begin{array}{l}\text { No. of amino } \\
\text { acids }\end{array}$} & \multicolumn{3}{|c|}{ Spectral counting } \\
\hline & & & & Non-cancer & Cancer & $\begin{array}{l}\text { Fold change } \\
\text { (Rsc) }\end{array}$ \\
\hline 1 & FCGBP_HUMAN & (Q9Y6R7) IgGFc-binding protein & 5405 & 15 & 1 & -3.307086 \\
\hline 2 & K1C10_HUMAN & (P13645) Keratin, type I cytoskeletal 10 & 584 & 7 & 0 & -3.163409 \\
\hline 3 & H3C_HUMAN & (Q6NXT2) Histone H3.3C & 135 & 12 & 1 & -3.006964 \\
\hline 4 & CO1Ā1_HUMAN & (P02452) Collagen alpha-1(I) chain & 1464 & 6 & 0 & -2.975121 \\
\hline 5 & K22E_HUMAN & (P35908) Keratin, type II cytoskeletal 2 epidermal & 639 & 5 & 0 & -2.759125 \\
\hline 6 & K2C6B_HUMAN & (P04259) Keratin, type II cytoskeletal 6B & 564 & 5 & 0 & -2.759125 \\
\hline 7 & H2B1A_HUMAN & (Q96A08) Histone H2B type 1-A & 127 & 9 & 1 & -2.630943 \\
\hline 8 & K1C14_HUMAN & (P02533) Keratin, type I cytoskeletal 14 & 472 & 4 & 0 & -2.505717 \\
\hline 9 & K1C17_HUMAN & (Q04695) Keratin, type I cytoskeletal 17 & 432 & 4 & 0 & -2.505717 \\
\hline 10 & K2C5_HUMAN & (P13647) Keratin, type II cytoskeletal 5 & 590 & 4 & 0 & -2.505717 \\
\hline 11 & CRIP1_HUMAN & (P50238) Cysteine-rich protein 1 & 77 & 3 & 0 & -2.198995 \\
\hline 12 & MUC2_HUMAN & (Q02817) Mucin-2 & 5179 & 3 & 0 & -2.198995 \\
\hline 13 & ATPA $\bar{H} U M A N$ & (P25705) ATP synthase subunit alpha, mitochondrial & 553 & 6 & 1 & -2.125742 \\
\hline 14 & K2C1B HUMAN & (Q7Z794) Keratin, type II cytoskeletal 1b & 578 & 13 & 4 & -1.887275 \\
\hline 15 & LMNA_HUMAN & (P02545) Prelamin-A/C & 664 & 10 & 3 & -1.84682 \\
\hline 16 & K1C9_HUMAN & (P35527) Keratin, type I cytoskeletal 9 & 623 & 15 & 5 & -1.827612 \\
\hline 17 & ACTN"4_HUMAN & (O43707) Alpha-actinin-4 & 911 & 2 & 0 & -1.810107 \\
\hline 18 & GLUC_-̄HUMAN & (P01275) Glucagon & 180 & 2 & 0 & -1.810107 \\
\hline 19 & RS7_HUMMAN & (P62081) 40S ribosomal protein S7 & 194 & 2 & 0 & -1.810107 \\
\hline 20 & K2C1_HUMAN & (P04264) Keratin, type II cytoskeletal 1 & 644 & 39 & 18 & -1.541287 \\
\hline 21 & CLCA1_HUMAN & (A8K7I4) Calcium-activated chloride channel regulator 1 & 914 & 7 & 3 & -1.393723 \\
\hline 22 & CAH1_-̄HUMAN & (P00915) Carbonic anhydrase 1 & 261 & 1 & 0 & -1.277731 \\
\hline 23 & GSLGī_HUMAN & (Q92896) Golgi apparatus protein 1 & 1179 & 1 & 0 & -1.277731 \\
\hline 24 & LAMB3_HUMAN & (Q13751) Laminin subunit beta-3 & 1172 & 1 & 0 & -1.277731 \\
\hline 25 & MK15_HUMAN & (Q8TD08) Mitogen-activated protein kinase 15 & 544 & 1 & 0 & -1.277731 \\
\hline 26 & RS30 HUMAN & (P62861) 40S ribosomal protein S30 & 59 & 1 & 0 & -1.277731 \\
\hline 27 & ZHAN̄G_HUMAN & (Q9NS37) CREB/ATF bZIP transcription factor & 354 & 1 & 0 & -1.277731 \\
\hline 28 & ZN408_HUMAN & (Q9H9D4) Zinc finger protein 408 & 720 & 1 & 0 & -1.277731 \\
\hline 29 & PGBM_HUMAN & $\begin{array}{l}\text { (P98160) Basement membrane-specific heparan sulfate } \\
\text { proteoglycan core protein }\end{array}$ & 4391 & 1 & 0 & -1.277731 \\
\hline 30 & DPYL2_HUMAN & (Q16555) Dihydropyrimidinase-related protein 2 & 572 & 1 & 0 & -1.277731 \\
\hline 31 & RL31_HUMAN & (P62899) 60S ribosomal protein L31 & 125 & 1 & 0 & -1.277731 \\
\hline 32 & HNRPL_HUMAN & (P14866) Heterogeneous nuclear ribonucleoprotein L & 589 & 1 & 0 & -1.277731 \\
\hline 33 & FLNA_HUMAN & (P21333) Filamin-A & 2647 & 1 & 0 & -1.277731 \\
\hline 34 & CO6A2_HUMAN & (P12110) Collagen alpha-2(VI) chain & 1019 & 1 & 0 & -1.277731 \\
\hline 35 & ARPC5_HUMAN & (O15511) Actin-related protein 2/3 complex subunit 5 & 151 & 1 & 0 & -1.277731 \\
\hline 36 & RS23_HUMAN & (P62266) 40S ribosomal protein S23 & 143 & 1 & 0 & -1.277731 \\
\hline 37 & MYH14_HUMAN & (Q7Z406) Myosin-14 & 1995 & 1 & 0 & -1.277731 \\
\hline 38 & EF2_HŪMAN & (P13639) Elongation factor 2 & 858 & 1 & 0 & -1.277731 \\
\hline 39 & ECHB HUMAN & (P55084) Trifunctional enzyme subunit beta, mitochondrial & 474 & 1 & 0 & -1.277731 \\
\hline 40 & FA48A- HUMAN & (Q8NEM7) Protein FAM48A & 779 & 1 & 0 & -1.277731 \\
\hline 41 & SETX $\bar{H} U M A N$ & (Q7Z333) Probable helicase senataxin & 2677 & 1 & 0 & -1.277731 \\
\hline 42 & GFAP_HUMAN & (P14136) Glial fibrillary acidic protein & 432 & 1 & 0 & -1.277731 \\
\hline 43 & PERI_HUMAN & (P41219) Peripherin & 470 & 1 & 0 & -1.277731 \\
\hline 44 & POTEE_HUMAN & (Q6S8J3) POTE ankyrin domain family member E & 1075 & 1 & 0 & -1.277731 \\
\hline 45 & TTHY_HUMAN & (P02766) Transthyretin & 147 & 1 & 0 & -1.277731 \\
\hline 46 & CAH2_HUMAN & (P00918) Carbonic anhydrase 2 & 260 & 1 & 0 & -1.277731 \\
\hline 47 & ENPL_HUMAN & (P14625) Endoplasmin & 803 & 1 & 0 & -1.277731 \\
\hline 48 & HS71L_HUMAN & (P34931) Heat shock $70 \mathrm{kDa}$ protein 1-like & 641 & 1 & 0 & -1.277731 \\
\hline 49 & CBR1_HUMAN & (P16152) Carbonyl reductase [NADPH] 1 & 277 & 1 & 0 & -1.277731 \\
\hline 50 & SPTA2_HUMAN & (Q13813) Spectrin alpha chain, brain & 2472 & 1 & 0 & -1.277731 \\
\hline 51 & HNRCĒ_HUMAN & (O60812) Heterogeneous nuclear ribonucleoprotein C-like 1 & 293 & 1 & 0 & -1.277731 \\
\hline 52 & UGGG1_HUMAN & (Q9NYU2) UDP-glucose:glycoprotein glucosyltransferase 1 & 1555 & 1 & 0 & -1.277731 \\
\hline 53 & OST48_HUMAN & $\begin{array}{l}\text { (P39656) Dolichyl-diphosphooligosaccharide-protein } \\
\text { glycosyltransferase } 48 \mathrm{kDa} \text { subunit }\end{array}$ & 456 & 1 & 0 & -1.277731 \\
\hline 54 & ACTA_HUMAN & (P62736) Actin, aortic smooth muscle & 377 & 40 & 23 & -1.238478 \\
\hline 55 & AGR2_HUMAN & (O95994) Anterior gradient protein 2 homolog & 175 & 4 & 2 & -1.124439 \\
\hline 56 & TBB2C_HUMAN & (P68371) Tubulin beta-2C chain & 445 & 12 & 7 & -1.124173 \\
\hline 57 & KCRB_HUMAN & (P12277) Creatine kinase B-type & 381 & 8 & 5 & -1.001492 \\
\hline 58 & HSP7C_HUMAN & (P11142) Heat shock cognate $71 \mathrm{kDa}$ protein & 643 & 1 & 5 & 1.051122 \\
\hline 59 & PPIA_HUMAN & (P62937) Peptidyl-prolyl cis-trans isomerase A & 165 & 1 & 5 & 1.051122 \\
\hline 60 & SBP1_HUMAN & (Q13228) Selenium-binding protein 1 & 472 & 1 & 5 & 1.051122 \\
\hline
\end{tabular}


Table 3 (continued)

\begin{tabular}{|c|c|c|c|c|c|c|}
\hline \multirow[t]{2}{*}{ No. } & \multirow[t]{2}{*}{ ID } & \multirow[t]{2}{*}{ Accession number and description } & \multirow{2}{*}{$\begin{array}{l}\text { No. of amino } \\
\text { acids }\end{array}$} & \multicolumn{3}{|c|}{ Spectral counting } \\
\hline & & & & Non-cancer & Cancer & $\begin{array}{l}\text { Fold change } \\
\text { (Rsc) }\end{array}$ \\
\hline 61 & G3P_HUMAN & (P04406) Glyceraldehyde-3-phosphate dehydrogenase & 335 & 6 & 19 & 1.0692919 \\
\hline 62 & ANXA2_HUMAN & (P07355) Annexin A2 & 339 & 1 & 6 & 1.2666356 \\
\hline 63 & MDHM_HUMAN & (P40926) Malate dehydrogenase, mitochondrial & 338 & 1 & 6 & 1.2666356 \\
\hline 64 & ALDOA_HUMAN & (P04075) Fructose-bisphosphate aldolase A & 364 & 0 & 3 & 1.3418115 \\
\hline 65 & LDHA_HUMAN & (P00338) L-lactate dehydrogenase A chain & 332 & 0 & 3 & 1.3418115 \\
\hline 66 & RL7_HUMMAN & (P18124) 60S ribosomal protein L7 & 248 & 0 & 3 & 1.3418115 \\
\hline 67 & S10Ā8_HUMAN & (P05109) Protein S100-A8 & 93 & 0 & 3 & 1.3418115 \\
\hline 68 & RL12_HUMAN & (P30050) 60S ribosomal protein L12 & 165 & 0 & 3 & 1.3418115 \\
\hline 69 & TBB1_HUMAN & (Q9H4B7) Tubulin beta-1 chain & 451 & 0 & 3 & 1.3418115 \\
\hline 70 & TAGL르UMAN & (P37802) Transgelin-2 & 199 & 0 & 3 & 1.3418115 \\
\hline 71 & LDHA_HUMAN & (P00338) L-lactate dehydrogenase A chain & 332 & 0 & 3 & 1.3418115 \\
\hline 72 & DHSA_HUMAN & $\begin{array}{l}\text { (P31040) Succinate dehydrogenase [ubiquinone] } \\
\text { flavoprotein subunit, mitochondrial }\end{array}$ & 664 & 0 & 3 & 1.3418115 \\
\hline 73 & S10A9_HUMAN & (P06702) Protein S100-A9 & 114 & 0 & 3 & 1.3418115 \\
\hline 74 & TBA1A_HUMAN & (Q71U36) Tubulin alpha-1 A chain & 451 & 0 & 3 & 1.3418115 \\
\hline 75 & FIBA_HUMAN & (P02671) Fibrinogen alpha chain & 866 & 0 & 4 & 1.6480523 \\
\hline 76 & CEAM5_HUMAN & $\begin{array}{l}\text { (P06731) Carcinoembryonic antigen-related cell } \\
\text { adhesion molecule } 5\end{array}$ & 702 & 0 & 4 & 1.6480523 \\
\hline 77 & RL1D1_HUMAN & (O76021) Ribosomal L1 domain-containing protein 1 & 490 & 0 & 4 & 1.6480523 \\
\hline 78 & 1433B_HUMAN & (P31946) 14-3-3 protein beta/alpha & 246 & 0 & 5 & 1.9009786 \\
\hline 79 & ACTN1_HUMAN & (P12814) Alpha-actinin-1 & 892 & 1 & 10 & 1.9060767 \\
\hline 80 & DEF1_HUMAN & (P59665) Neutrophil defensin 1 & 94 & 1 & 13 & 2.2513044 \\
\hline 81 & CH60_HUMAN & (P10809) $60 \mathrm{kDa}$ heat shock protein, mitochondrial & 573 & 2 & 23 & 2.5000084 \\
\hline 82 & H2B1C_HUMAN & (P62807) Histone H2B type 1-C/E/F/G/I & 126 & 0 & 9 & 2.620238 \\
\hline 83 & H31_HÜMAN & (P68431) Histone H3.1 & 136 & 0 & 13 & 3.1011611 \\
\hline 84 & TBB5_HUMAN & (P07437) Tubulin beta chain & 444 & 0 & 13 & 3.1011611 \\
\hline
\end{tabular}

Expression levels of these 84 proteins were more than two-fold higher or lower in cancer regions compared to non-cancer regions of CRC samples

\section{CRC cell lines}

CRC cell lines DLD-1, SW480, and SW620 and normal human colon epithelial cell line CCD $841 \mathrm{CoN}$ were purchased from the American Type Culture Collection (Manassas, VA, USA). All cells were cultured in RPMI 1640 medium supplemented with $10 \%$ fetal bovine serum (FBS) (Gibco, Carlsbad, CA, USA) in an atmosphere containing $5 \% \mathrm{CO}_{2}$.

\section{Protein preparation}

CRC cells were plated at a density of $5 \times 10^{5}$ cells per dish in a $100-\mathrm{mm}$ dish and grown in culture medium. After $72 \mathrm{~h}$, cells were solubilized in urea lysis buffer ( $7 \mathrm{M}$ urea, $2 \mathrm{M}$ thiourea, $5 \%$ CHAPS, and $1 \%$ Triton X-100). To quantify secreted protein, the culture medium of CRC cells was collected after $72 \mathrm{~h}$.

\section{Western blot analysis}

The cell extract, culture medium, and commercial human normal serum (ImmunoBioScience Corp., Mukilteo, WA) were subjected to SDS-PAGE under reducing conditions.
The separated proteins were transferred to polyvinylidene fluoride transfer membranes. Membranes were incubated with an anti-annexin A2 rabbit monoclonal antibody, antialdolase A rabbit monoclonal antibody, or anti-cyclophilin A antibody (Cell Signaling Technology Inc., Beverly, MA, USA) at $4{ }^{\circ} \mathrm{C}$ overnight. Membranes were then washed and incubated with HRP-conjugated anti-rabbit IgG antibody (American Qualex, San Clemente, CA). After washing, blots were visualized by enhanced chemiluminescence and detected using a myECL Imager system (ThermoFisher Scientific). The same membranes were reprobed with anti- $\beta$-actin antibody (Sigma) to confirm equal loading of the proteins. All Western blot analyses were performed three times.

\section{Statistical analysis}

All data are presented as the mean \pm standard error of the mean. Data between two groups were compared with the unpaired $t$ test. Values of $* P<0.05$ and $* * P<0.001$ were considered significant in all analyses. Computations were performed with GraphPad Prism version 5 (GraphPad Software, La Jolla, CA, USA). 
Fig. 4 Analysis of GO cellular components of identified proteins. Protein assignments to GO cellular component categories are shown only for significant categories $(p<0.05)$

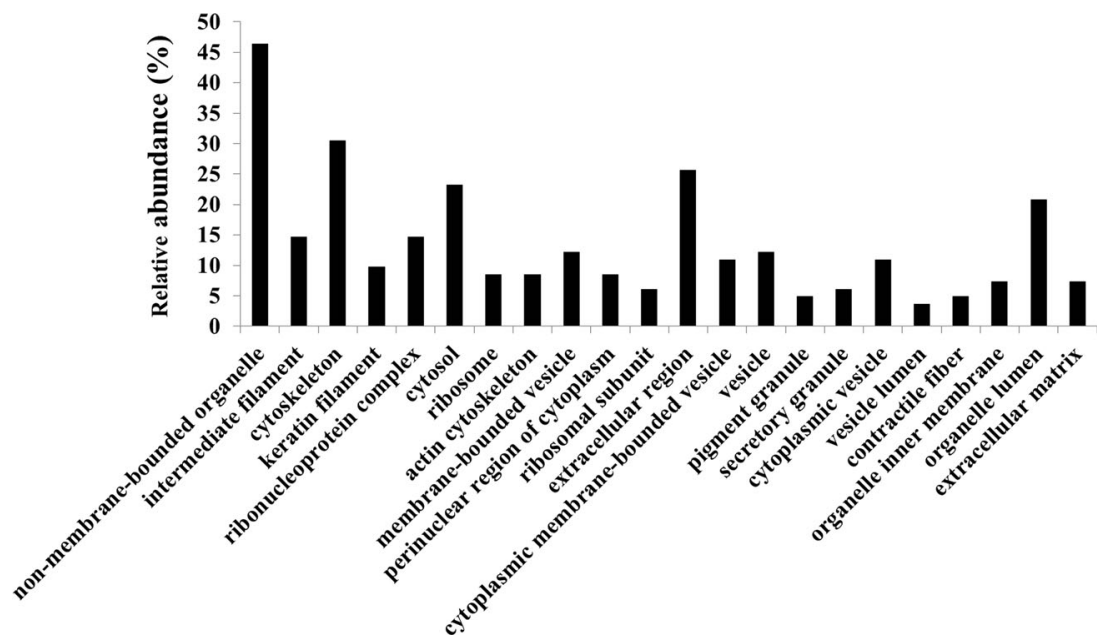

\section{Results}

\section{Protein identification and profiles in cancer and non-cancer regions of $\mathrm{CRC}$ tissue}

To investigate the molecular profile of proteins expressed in relation to cancer progression, we performed shotgun proteomic analysis using FFPE CRC tissue. We used manual dissection to separate the cancer regions and non-cancer regions from FFPE CRC tissues and examined the protein expression not only in cells but also in the stromal tissue surrounding cells (Fig. 1). We successfully identified proteins in both cancer and non-cancer regions of FFPE tissues (Table 1). Figure 2 shows the Venn map for the proteins identified.

\section{Semiquantitative comparison of proteins identified in cancer and non-cancer regions of CRC tissue}

We next performed a label-free semiquantitative method based on spectral counting to identify proteins with modified
Table 4 Proteins categorized as extracellular region proteins

\begin{tabular}{llc} 
No. & Accession number and description & Fold change (Rsc) \\
\hline 1 & (Q9Y6R7) IgGFc-binding protein & -3.3070855 \\
2 & (P02452) Collagen alpha-1(I) chain & -2.9751214 \\
3 & (Q02817) Mucin-2 & -2.1989951 \\
4 & (O43707) Alpha-actinin-4 & -1.8101074 \\
5 & (P01275) Glucagon & -1.8101074 \\
6 & (A8K7I4) Calcium-activated chloride channel regulator 1 & -1.3937233 \\
7 & (Q13751) Laminin subunit beta-3 & -1.2777305 \\
8 & (Q8TD08) Mitogen-activated protein kinase 15 & -1.2777305 \\
9 & (P98160) Basement membrane-specific heparan sulfate proteoglycan core protein & -1.2777305 \\
10 & (P21333) Filamin-A & -1.2777305 \\
11 & (P12110) Collagen alpha-2(VI) chain & -1.2777305 \\
12 & (P02766) Transthyretin & -1.2777305 \\
13 & (P00918) Carbonic anhydrase 2 & -1.2777305 \\
14 & (O95994) Anterior gradient protein 2 homolog & -1.1244392 \\
15 & (P62937) Peptidyl-prolyl cis-trans isomerase A & 1.05112197 \\
16 & (P07355) Annexin A2 & 1.26663565 \\
17 & (P04075) Fructose-bisphosphate aldolase A & 1.34181152 \\
18 & (P02671) Fibrinogen alpha chain & 1.64805231 \\
19 & (P12814) Alpha-actinin-1 & 1.90607666 \\
20 & (P59665) Neutrophil defensin 1 & 2.25130442 \\
21 & (P10809) 60 kDa heat shock protein, mitochondrial & 2.50000843 \\
\hline & &
\end{tabular}

Twenty-one proteins that were differentially expressed in cancer regions of CRC samples were classified as extracellular region proteins by gene ontology analysis 

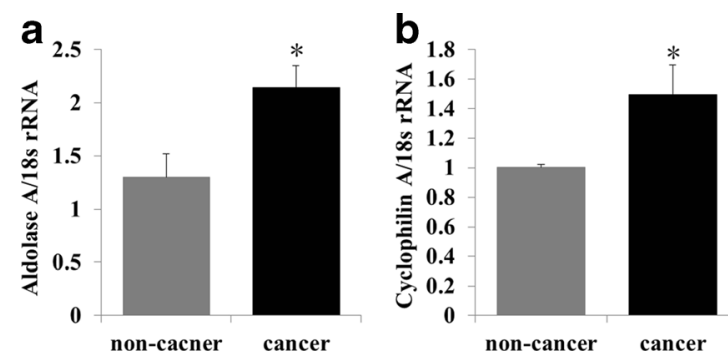

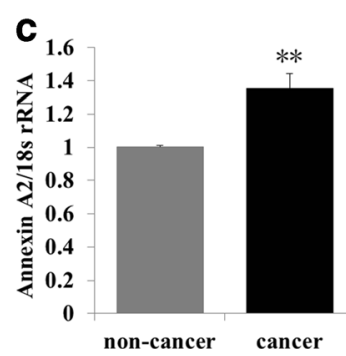

were significantly higher in cancer compared to non-cancer regions $(\mathbf{a}-\mathbf{c})$ $* p<0.05, * * p<0.001$

antigen-related cell adhesion molecule 5 (CEAM5, also known as CEA). By contrast, expression levels of housekeeping proteins such as $\beta$-actin and histone $\mathrm{H} 4$ did not change.

\section{Functional annotation of differentially expressed proteins in cancer regions of CRC tissue}

DAVID was used to perform GO analyses for the differentially expressed proteins identified for each cellular component (Fig. 4). Functional annotations were counted by normalizing to the total number of identified proteins. Based on the cellular component classification, we focused on 21 proteins classified in the extracellular region, which could potentially be detected in the blood of patients with CRC (Table 4).
Fig. 6 IHC validation of spectral counting results. Expression levels of aldolase A, cyclophilin $\mathrm{A}$, and annexin A2 were validated by IHC. Representative images of non-cancer regions (a, $\mathbf{d}$, and $\mathbf{g}$ ) and cancer regions (b, e, and $\mathbf{h})$. Graphs of IHC scores are shown in (c), (f), and (i). Cancer regions showed significantly stronger expression of these three proteins, which is consistent with spectral counting data. $* p<0.05$;

$* * p<0.001$. Original magnification, $100 \times$; insets, $400 \times$

\section{Aldolase A}

a

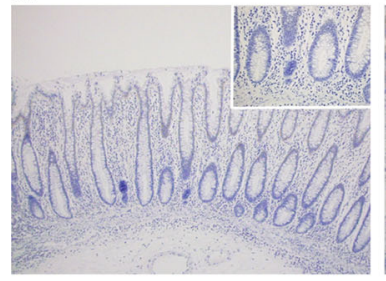

Cyclophilin A

d

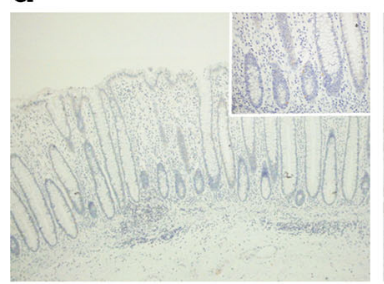

Annexin A2

g

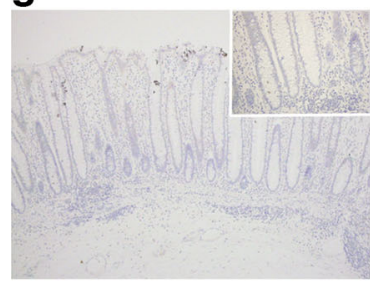

b

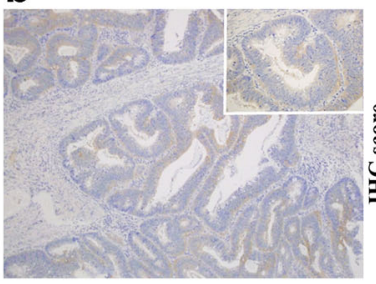

e

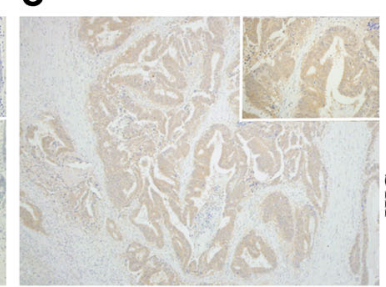

h

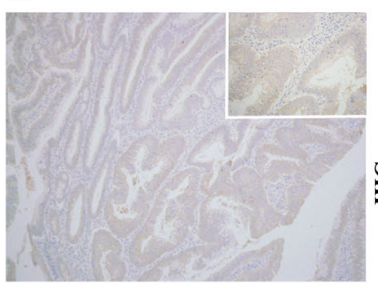

C

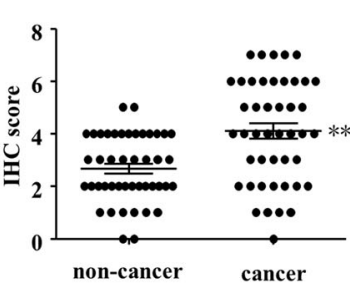

f

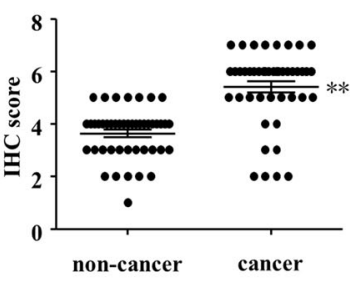

i

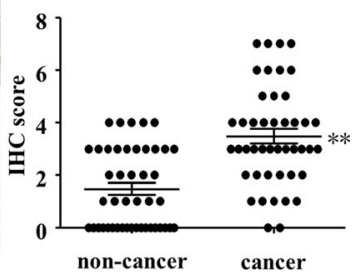


qRT-PCR analysis of aldolase $\mathrm{A}$, cyclophilin $\mathrm{A}$, and annexin $\mathrm{A} 2$ in cancer and non-cancer regions of $\mathrm{CRC}$ tissue

Fructose-bisphosphate aldolase A (aldolase A), peptidylprolyl cis-trans isomerase A (also known as cyclophilin A), and annexin A2 were upregulated in cancer regions (Table 3) and consequently selected as candidate diagnostic biomarkers. To confirm the higher expression levels of the candidate proteins in cancer regions compared to noncancer regions, we performed qRT-PCR analysis. Expression levels of the mRNAs for these proteins were significantly higher in cancer compared to non-cancer regions (Fig. 5a-c).

\section{IHC analysis of aldolase A, cyclophilin A, and annexin A2 in cancer and non-cancer regions of $\mathrm{CRC}$ tissue}

We next performed IHC analysis of the three candidate proteins for validation with tissues from $45 \mathrm{CRC}$ cases (Table 2). IHC analysis revealed that expression levels of all three proteins were significantly higher in cancer regions compared to non-cancer regions (Fig. 6).

\section{Expression of aldolase A, cyclophilin A, and annexin A2 in CRC cell lines}

To investigate whether the candidate proteins were suitable as biomarkers, their mRNA and protein expression levels in CRC cell lines were determined by qRT-PCR analysis and western blotting. All tested cell lines expressed the three candidate proteins, and their expression levels in CRC cells tended to be higher than in normal colon epithelial cells (Fig. 7a-d). Aldolase A was not detected in the culture medium of the three CRC cell lines, but it was detected in normal colon epithelial cells (Fig. 7e, upper panel). Cyclophilin A was not detected in the culture medium of any cell lines (Fig. 7e, middle panel). The level of annexin A2 in the culture medium appeared to depend on the level of annexin A2 in the cell extract for each cell line (Fig. 7e, lower panel). Finally, we detected aldolase A in normal human serum and confirmed that the level depended on the loaded serum volume (Fig. 7f, lane 1-3).

\section{Discussion}

In this study, we used a gel-free LC-MS-based proteomics approach to identify potential biomarkers for cancer in FFPE
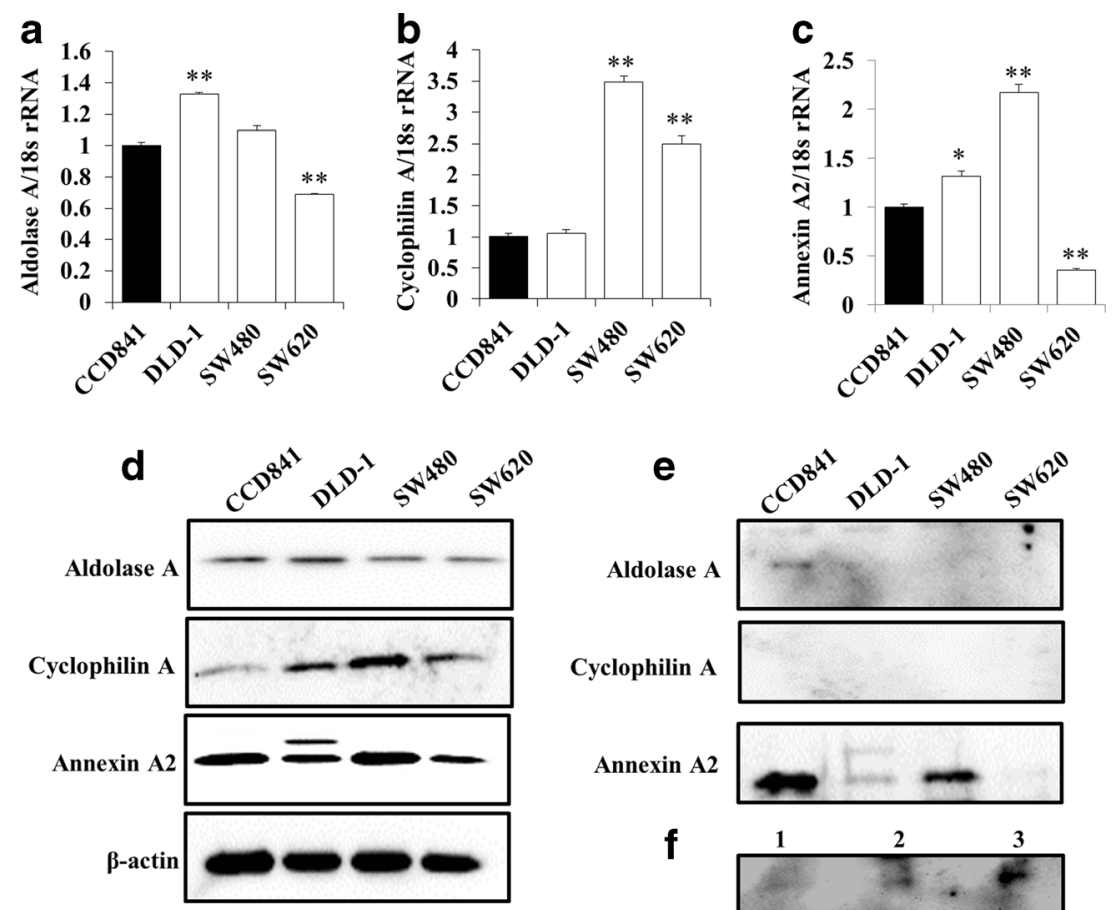

Fig. 7 Expression of the three candidate proteins in CRC cell lines. Expression levels of aldolase A, cyclophilin A, and annexin A2 were examined by qRT-PCR and western blot. Expression levels of aldolase A (a), cyclophilin A (b), and annexin A2 (c) mRNA in CRC cells were almost all significantly higher than in normal colon epithelium. $* p<0.05$; $* * p<0.001$. Expression levels of the three candidate proteins in cell extracts from CRC cell lines were similar to mRNA expression levels (d). Aldolase A was only detected in the culture medium of normal colon

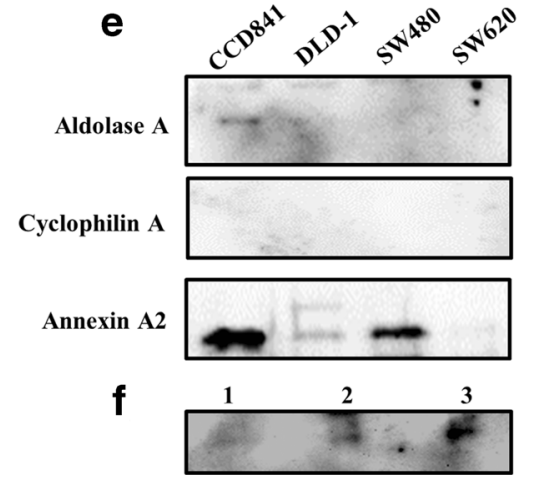

epithelium (e, upper panel). Cyclophilin A was not detected in any of the examined culture medium samples (e, middle panel). The level of annexin A2 in the culture medium appeared to depend on the level in the cell extract for each cell line (e, lower panel). Aldolase A was detected in human normal serum, and the level of aldolase A detected depended on the volume of serum loaded (f, lane 1, $10 \mu \mathrm{g}$ serum; lane 2, $20 \mu \mathrm{g}$ serum; lane 3, $30 \mu \mathrm{g}$ serum) 
CRC tissues. We used semiquantitative methods based on spectral counting to detect 84 proteins in which expression levels were altered $>2$-fold in cancer compared to noncancer regions of CRC tissue (Table 3). CEA, a known tumor marker used in diagnostic blood tests for CRC, was among these proteins. Thus, the approach used in this study could potentially be used to identify novel biomarker candidates.

To identify early detection markers for CRC that can be detected by diagnostic blood tests, we focused on proteins in the "extracellular region" category of cellular components based on the results of GO analysis (Table 4). These proteins are secreted into the extracellular space and thus may potentially be detected in blood. These criteria led us to select aldolase A, cyclophilin A, and annexin A2 as candidates. We did not include HSP60, neutrophil defensin1, or alpha-actinin1 , because previous reports had already suggested that these proteins might be biomarkers for CRC [26-29].

Validation studies revealed that mRNA and protein expression levels of the three candidate proteins were significantly higher in the cancer region compared to non-cancer regions (Figs. 5 and 6). Moreover, to evaluate whether these proteins were useful as biomarkers, we also investigated their mRNA and protein expression levels in CRC cell lines and secretion of the proteins into the medium. Cyclophilin A was not secreted into the culture medium of all tested CRC cell lines (Fig. 7e). Thus, cyclophilin A is not suitable as a diagnostic blood tumor marker. On the other hand, annexin A2 expression in SW480 cells derived from the primary tumor site of a CRC patient was higher than expression in SW620 cells that were established from a metastatic site in the same patient (Fig. 7c-e). Decreased annexin A2 expression in cancer cells could play a role in the progression and metastasis of CRC and may be useful for predicting metastasis.

Although mRNA and protein expression of aldolase A were detected in all tested cell lines (Fig. 7a and d), aldolase A protein was not detected in the culture medium of the three CRC cell lines, while it was clearly detected in the culture medium of normal colon epithelial cells (Fig. 7e). Moreover, aldolase A was detected in normal human serum (Fig. 7f). These results suggest that secretion of aldolase A may be suppressed by dysfunction of aldolase A due to accumulated genetic mutations during the carcinogenesis process of CRC. Therefore, decreased aldolase A levels in blood may be useful as a biomarker for the early diagnosis of CRC.

Aldolase A is a glycolytic enzyme and contributes to various cellular functions related to muscle maintenance, cell shape and mobility regulation, striated muscle contraction, actin filament organization, and ATP biosynthesis [30-40]. There are several reports of elevated expression of aldolase A in the serum of patients with malignant tumors, such as those with lung and renal cancer $[41,42]$. However, the expression kinetics and functions of aldolase A in CRC are not well understood. To our knowledge, this is the first report of decreased secretion of aldolase A in CRC. Thus, further studies are necessary to clarify the decrease in aldolase A levels in the blood of CRC patients in order to determine if this protein can be used as an early detection biomarker for CRC.

\section{Conclusion}

In conclusion, we identified 248 proteins from FFPE CRC tissues using global shotgun proteomics. A label-free semiquantitative method based on spectral counting and GO identified 21 candidate early detection biomarkers for CRC that could potentially be detected in blood. Validation studies revealed that cyclophilin A, annexin A2, and aldolase A expression levels were significantly higher in cancer compared to non-cancer regions. In vitro studies showed that secretion of aldolase A into the culture medium was clearly suppressed in CRC cancer cells to normal colon epithelium. Finally, aldolase A may play an important role in the carcinogenesis process of CRC and may be useful as an early detection biomarker for CRC.

Acknowledgments We thank Mr. K. Teduka and Mr. T. Fujii for technical assistance (Department of Integrated Diagnostic Pathology). This work was supported by a Grant-in-Aid for Scientific Research (C, No. 15 K09054 to T. Yamamoto) and also in part by the MEXT-Supported Program of the Strategic Research Foundation at Private Universities (2014-2018) (S1411037)

\section{Compliance with ethical standards}

Conflicts of interest None.

Open Access This article is distributed under the terms of the Creative Commons Attribution 4.0 International License (http:// creativecommons.org/licenses/by/4.0/), which permits unrestricted use, distribution, and reproduction in any medium, provided you give appropriate credit to the original author(s) and the source, provide a link to the Creative Commons license, and indicate if changes were made.

\section{References}

1. Kuusela P, Jalanko H, Roberts P, Sipponen P, Mecklin JP, Pitkanen R, Makela O. Comparison of CA 19-9 and carcinoembryonic antigen (CEA) levels in the serum of patients with colorectal diseases. Br J Cancer. 1984;49:135-9.

2. Zamcheck N, Pusztaszeri G. CEA, AFP and other potential tumor markers. CA Cancer J Clin. 1975;25:204-14.

3. Shi SR, Cote RJ, Taylor CR. Antigen retrieval immunohistochemistry: past, present, and future. The journal of histochemistry and cytochemistry: official journal of the Histochemistry Society. 1997;45:327-43.

4. Tourtellotte WW, Verity AN, Schmid P, Martinez S, Shapshak P. Covalent binding of formalin fixed paraffin embedded brain tissue 
sections to glass slides suitable for in situ hybridization. J Virol Methods. 1987;15:87-99.

5. Zhao J, Wu R, Au A, Marquez A, Yu Y, Shi Z. Determination of HER2 gene amplification by chromogenic in situ hybridization (CISH) in archival breast carcinoma. Modern pathology: an official journal of the United States and Canadian Academy of Pathology, Inc. 2002;15:657-65.

6. D.A. Prieto, B.L. Hood, M.M. Darfler, T.G. Guiel, D.A. Lucas, T.P. Conrads, T.D. Veenstra, D.B. Krizman, Liquid tissue: proteomic profiling of formalin-fixed tissues. BioTechniques Suppl. 2005. 32-35.

7. Hood BL, Darfler MM, Guiel TG, Furusato B, Lucas DA, Ringeisen BR, Sesterhenn IA, Conrads TP, Veenstra TD, Krizman DB. Proteomic analysis of formalin-fixed prostate cancer tissue. Molecular \& cellular proteomics: MCP. 2005;4:1741-53.

8. Hood BL, Conrads TP, Veenstra TD. Unravelling the proteome of formalin-fixed paraffin-embedded tissue. Briefings in functional genomics \& proteomics. 2006;5:169-75.

9. Hood BL, Conrads TP, Veenstra TD. Mass spectrometric analysis of formalin-fixed paraffin-embedded tissue: unlocking the proteome within. Proteomics. 2006;6:4106-14.

10. Guo T, Wang W, Rudnick PA, Song T, Li J, Zhuang Z, Weil RJ, DeVoe DL, Lee CS, Balgley BM. Proteome analysis of microdissected formalin-fixed and paraffin-embedded tissue specimens. The journal of histochemistry and cytochemistry : official journal of the Histochemistry Society. 2007;55:763-72.

11. Kislinger T, Cox B, Kannan A, Chung C, Hu P, Ignatchenko A, Scott MS, Gramolini AO, Morris Q, Hallett MT, Rossant J, Hughes TR, Frey B, Emili A. Global survey of organ and organelle protein expression in mouse: combined proteomic and transcriptomic profiling. Cell. 2006;125:173-86.

12. Nirmalan NJ, Harnden P, Selby PJ, Banks RE. Mining the archival formalin-fixed paraffin-embedded tissue proteome: opportunities and challenges. Mol BioSyst. 2008;4:712-20.

13. Scicchitano MS, Dalmas DA, Boyce RW, Thomas HC, Frazier KS. Protein extraction of formalin-fixed, paraffin-embedded tissue enables robust proteomic profiles by mass spectrometry. The journal of histochemistry and cytochemistry : official journal of the Histochemistry Society. 2009;57:849-60.

14. Sprung Jr RW, Brock JW, Tanksley JP, Li M, Washington MK, Slebos RJ, Liebler DC. Equivalence of protein inventories obtained from formalin-fixed paraffin-embedded and frozen tissue in multidimensional liquid chromatography-tandem mass spectrometry shotgun proteomic analysis. Molecular \& cellular proteomics: MCP. 2009;8:1988-98.

15. Naidoo K, Jones R, Dmitrovic B, Wijesuriya N, Kocher H, Hart IR, Crnogorac-Jurcevic T. Proteome of formalin-fixed paraffin-embedded pancreatic ductal adenocarcinoma and lymph node metastases. J Pathol. 2012;226:756-63.

16. Paulo JA, Lee LS, Banks PA, Steen H, Conwell DL. Proteomic analysis of formalin-fixed paraffin-embedded pancreatic tissue using liquid chromatography tandem mass spectrometry. Pancreas. 2012;41:175-85.

17. Jiang X, Feng S, Tian R, Ye M, Zou H. Development of efficient protein extraction methods for shotgun proteome analysis of formalin-fixed tissues. J Proteome Res. 2007;6:1038-47.

18. Hamilton SR, Aaltonen LA. Pathology and genetics of tumours of the digestive system. In: Kleihues P, Sobin LH, editors. World health organization classification of tumours. Lyon: IARCPress; 2000.

19. Bluemlein K, Ralser M. Monitoring protein expression in wholecell extracts by targeted label- and standard-free LC-MS/MS. Nat Protoc. 2011;6:859-69.
20. Kawamura T, Nomura M, Tojo H, Fujii K, Hamasaki H, Mikami S, Bando Y, Kato H, Nishimura T. Proteomic analysis of lasermicrodissected paraffin-embedded tissues: (1) stage-related protein candidates upon non-metastatic lung adenocarcinoma. J Proteome. 2010;73:1089-99.

21. Old WM, Meyer-Arendt K, Aveline-Wolf L, Pierce KG, Mendoza A, Sevinsky JR, Resing KA, Ahn NG. Comparison of label-free methods for quantifying human proteins by shotgun proteomics. Molecular \& cellular proteomics : MCP. 2005;4:1487-502.

22. Zybailov B, Coleman MK, Florens L, Washburn MP. Correlation of relative abundance ratios derived from peptide ion chromatograms and spectrum counting for quantitative proteomic analysis using stable isotope labeling. Anal Chem. 2005;77:6218-24.

23. Dennis Jr G, Sherman BT, Hosack DA, Yang J, Gao W, Lane HC, Lempicki RA. DAVID: database for annotation, visualization, and integrated discovery. Genome Biol. 2003;4:P3.

24. da Huang W, Sherman BT, Lempicki RA. Systematic and integrative analysis of large gene lists using DAVID bioinformatics resources. Nat Protoc. 2009;4:44-57.

25. da Huang W, Sherman BT, Lempicki RA. Bioinformatics enrichment tools: paths toward the comprehensive functional analysis of large gene lists. Nucleic Acids Res. 2009;37:1-13.

26. Hamelin C, Cornut E, Poirier F, Pons S, Beaulieu C, Charrier JP, Haidous H, Cotte E, Lambert C, Piard F, Ataman-Onal Y, ChoquetKastylevsky G. Identification and verification of heat shock protein 60 as a potential serum marker for colorectal cancer. The FEBS journal. 2011;278:4845-59.

27. van den Broek I, Sparidans RW, Engwegen JY, Cats A, Depla AC, Schellens JH, Beijnen JH. Evaluation of human neutrophil peptide$1,-2$ and -3 as serum markers for colorectal cancer. Cancer biomarkers : section A of Disease markers. 2010;7:109-15.

28. Kemik O, Kemik AS, Sumer A, Begenik H, Purisa S, Tuzun S. Human neutrophil peptides 1, 2 and 3 (HNP 1-3): elevated serum levels in colorectal cancer and novel marker of lymphatic and hepatic metastasis. Human \& experimental toxicology. 2013;32:167-71.

29. Thorsen K, Sorensen KD, Brems-Eskildsen AS, Modin C, Gaustadnes M, Hein AM, Kruhoffer M, Laurberg S, Borre M, Wang K, Brunak S, Krainer AR, Torring N, Dyrskjot L, Andersen CL, Orntoft TF. Alternative splicing in colon, bladder, and prostate cancer identified by exon array analysis. Molecular \& cellular proteomics : MCP. 2008;7:1214-24.

30. Kusakabe T, Motoki K, Hori K. Mode of interactions of human aldolase isozymes with cytoskeletons. Arch Biochem Biophys. 1997;344:184-93.

31. Harris SJ, Winzor DJ. Enzyme kinetic evidence of active-site involvement in the interaction between aldolase and muscle myofibrils. Biochim Biophys Acta. 1987;911:121-6.

32. Carr D, Knull H. Aldolase-tubulin interactions: removal of tubulin C-terminals impairs interactions. Biochem Biophys Res Commun. 1993;195:289-93.

33. St-Jean M, Izard T, Sygusch J. A hydrophobic pocket in the active site of glycolytic aldolase mediates interactions with WiskottAldrich syndrome protein. J Biol Chem. 2007;282:14309-15.

34. Arnold H, Pette D. Binding of glycolytic enzymes to structure proteins of the muscle. European journal of biochemistry/FEBS. 1968;6:163-71.

35. Walsh JL, Knull HR. Heteromerous interactions among glycolytic enzymes and of glycolytic enzymes with F-actin: effects of poly (ethylene glycol). Biochim Biophys Acta. 1988;952:83-91.

36. Clarke FM, Masters CJ. On the association of glycolytic enzymes with structural proteins of skeletal muscle. Biochim Biophys Acta. 1975;381:37-46. 
37. Knull HR, Walsh JL. Association of glycolytic enzymes with the cytoskeleton. Curr Top Cell Regul. 1992;33:15-30.

38. Walsh JL, Keith TJ, Knull HR. Glycolytic enzyme interactions with tubulin and microtubules. Biochim Biophys Acta. 1989;999:64-70.

39. Tochio T, Tanaka H, Nakata S, Hosoya H. Fructose-1,6bisphosphate aldolase a is involved in $\mathrm{HaCaT}$ cell migration by inducing lamellipodia formation. J Dermatol Sci. 2010;58:123-9.

40. Lu M, Holliday LS, Zhang L, Dunn Jr WA, Gluck SL. Interaction between aldolase and vacuolar $\mathrm{H}+-$ ATPase: evidence for direct coupling of glycolysis to the ATP-hydrolyzing proton pump. J Biol Chem. 2001;276:30407-13.

41. Asaka M, Kimura T, Meguro T, Kato M, Kudo M, Miyazaki T, Alpert E. Alteration of aldolase isozymes in serum and tissues of patients with cancer and other diseases. J Clin Lab Anal. 1994;8: 144-8.

42. Takashi M, Zhu Y, Nakano Y, Miyake K, Kato K. Elevated levels of serum aldolase a in patients with renal cell carcinoma. Urol Res. 1992;20:307-11. 